\title{
Suicidal behavior and antiepileptic drugs in epilepsy: analysis of the emerging evidence
}

This article was published in the following Dove Press journal:

Drug, Healthcare and Patient Safety

I5 June 201 I

Number of times this article has been viewed

\author{
Marco Mula' \\ Dale C Hesdorffer ${ }^{2}$ \\ 'Department of Clinical and \\ Experimental Medicine, Amedeo \\ Avogadro University and Division \\ of Neurology, University Hospital \\ Maggiore della Carità, Novara, \\ Italy; ${ }^{2}$ Gertrude H Sergievsky Center \\ and Department of Epidemiology, \\ Columbia University, New York, \\ NY, USA
}

\begin{abstract}
Two years after the warning issued by the Food and Drug Administration on an increased risk of suicide for people taking antiepileptic drugs (AEDs), a number of pharmacoepidemiologic studies have been published but the scientific community is far from definitive answers. The present paper is aimed at reviewing available evidence on the association between AEDs and suicidal behavior, discussing major variables involved such as the relationship between epilepsy, depression, and suicide and the psychotropic potential of AEDs. All studies published so far show a lack of concordance and are constrained by various methodological limitations. What seems to be established is that mood disorders represent a frequent comorbidity in epilepsy and suicide is a serious complication more frequently encountered in epilepsy rather than in the general population. Moreover, a subgroup of patients appears to be at risk of developing treatment-emergent psychiatric adverse effects of AEDs independently of the specific mechanism of action of the drug. The prior history of suicide attempt, especially preceding the onset of the epilepsy, may represent a key element explaining why what is observed is independent of the specific mechanism of the drug. In general terms, risks associated with stopping, or not even starting, AEDs in epilepsy might well be in excess of the risk of suicide in epilepsy, as deaths due to accident and epilepsy itself may predominate. Clinicians need to pay attention not only to seizure patterns when choosing the appropriate AED but also to a number of different parameters (eg, age, gender, working needs, medical comorbidities, history of psychiatric disorders, and suicidality before epilepsy onset) and not the least the mental state of the patient. Missing severe complications such as suicidal behavior or delaying its treatment may worsen the prognosis of epilepsy.
\end{abstract}

Keywords: antiepileptic drugs, suicide, depression, epilepsy, FDA

\section{Introduction}

The question of whether people taking antiepileptic drugs (AEDs) are at increased risk of suicidal behavior has been a matter of debate in the scientific community for more than 2 years. ${ }^{1-4}$ In fact, in 2008, the Food and Drug Administration (FDA) issued a warning to health care professionals about such increased risk. ${ }^{5}$ Pharmaceutical companies had been previously asked to submit data from placebo controlled trials of AEDs, regardless of indication, with at least 30 patients involved. Spontaneously reported suicidal behaviors occurring during double-blind trials with an AED (or within 1 day of stopping) were sought and categorized. Data were provided on the use of 11 AEDs used for epilepsy (25\% patients), psychiatric indications ( $27 \%$ patients), and 'other conditions' ( $48 \%$ patients). In the main analysis, almost 28,000 people taking AED and over 16,000 people taking placebo were considered. There were 4 completed
Correspondence: Marco Mula Division of Neurology, University Hospital Maggiore della Carità, C.so Mazzini 18, 28I00 Novara, Italy Tel +39032I 373337|

Fax +39 032I 3733298

Email marco.mula@med.unipmn.it 
suicides altogether, all in people taking AEDs and none in those taking placebo. The overall odds ratio (OR) for the association between AEDs and suicidal behavior or ideation was 1.8 (95\% CI 1.24 to 2.66$)$, but it was not statistically significantly high for ideation alone [1.45 (95\% CI 0.93 to 2.3)]. ${ }^{6}$ As a result of the analysis, the FDA required that all manufacturers of drugs in this class include a warning in their labeling and develop a medication guide for patients, informing them of the risks of suicidal thoughts or actions. The FDA also suggested that the warning language be carefully worded and that it mention the risk of not treating the underlying condition. ${ }^{7}$

Different authors have pointed out that the FDA's concern might have been excessive, ${ }^{3,4}$ and that the risk of stopping (or not starting) AEDs, particularly in people with epilepsy, would be far greater than this hypothetic small increased risk of suicidal behavior. ${ }^{8}$ Nevertheless, the strictly limited data on the subject has led to the publication of a number of pharmacoepidemiologic studies during the last year. The present paper is aimed at reviewing and discussing emerging evidence on the association between AEDs and suicidality, taking into account a number of other issues that are essential to the understanding of the problem such as comorbidities in epilepsy, history of psychiatric disorders and suicidal behavior predating the onset of epilepsy, and the psychotropic potential of AEDs.

\section{Epilepsy, depression, and suicide: what is the role of AEDs?}

Mood disorders represent the most frequent psychiatric comorbidity in patients with epilepsy and reasons for such a close link are both biological and psychosocial. ${ }^{9}$ Community-based studies in unselected sample of patients with epilepsy, such as the General Practice study ${ }^{10}$ and the Community Health Survey, ${ }^{11}$ reported a prevalence rate for depressive disorders in the order of $20 \%$ to $22 \%$. It is evident that this rate is much higher in epilepsy than rates reported in the general population - about $12 \%$. Studies from selected patient groups, such as tertiary referral centers or from surgery programs, noted an even higher prevalence of depression, ranging between $30 \%$ to $50 \%{ }^{12,13}$ Such differences partially reflect the severity of the seizure disorder. In fact, while depression seems to occur in only $4 \%$ of seizure-free patients, it seems very common, up to $21 \%$, in patients with uncontrolled epilepsy, ${ }^{14}$ suggesting that, indeed, patients with epilepsy and continuing seizures are significantly more likely to suffer from depression than those in remission. Whether this may be due to a biological underpinning or to the psychosocial limitations associated with a severe seizure disorders or both has not yet been clarified.

A number of publications suggest that not only depression but also suicide is overrepresented in epilepsy. A metaanalysis of 12 studies estimated the Standardized Mortality Ratio (SMR) for suicide in people with epilepsy as 5.1 (95\% CI 3.9 to 6.6), suggesting that people with epilepsy are more than 5 times as likely to commit suicide than the general population. ${ }^{15}$ In addition, the authors found the risk of suicide to be high in people with surgically treated epilepsy. A more recent, large meta-analysis, found the SMR to be 3.3 (95\% CI 2.8 to 3.7$).{ }^{16}$ The SMR was again found to be increased in people with surgically treated epilepsy. Several studies have attempted to establish risk factors for suicide in people with epilepsy. In the general population, about $90 \%$ of people who successfully commit suicide have at least one psychiatric disorder at the time. ${ }^{17}$ Epilepsy is frequently associated with psychiatric comorbidity, but it is unlikely that the psychiatric comorbidity is the only element responsible for the increase in suicides in people with epilepsy. A study using data sources from Denmark investigated over 20,000 people who died from suicide and over 400,000 controls (up to 20 for each case) alive on the date of the suicide and matched for age and sex. ${ }^{18}$ Of those who committed suicide, almost 500 had epilepsy. Those with epilepsy were more than 3 times more likely to commit suicide than those without epilepsy. More people with epilepsy had psychiatric disease (rate ratio 4.3), but the rate ratio of suicide in people with epilepsy was still 2 compared with those without epilepsy after excluding those with psychiatric disease and adjusting for various factors. Some authors have suggested that the rate of suicide is higher in people with temporal lobe epilepsy. ${ }^{15}$ Idiopathic generalized epilepsy has recently been studied with both retrospective and prospective methods in 145 consecutive adults. ${ }^{19}$ No epilepsy-related factors were associated with suicide attempts while all index cases had a current psychiatric disorder (mood disorders, neurotic disorders, or personality disorders) and psychosocial events were considerably relevant. The risk factors for suicide in epilepsy are still far from being elucidated. This relationship is likely to be multifactorial in origin with biological, constitutional, and psychosocial variables being implicated. At any rate, it seems evident that suicide prevention represents a relevant issue in epilepsy and careful attention needs to be paid in selected cases.

In this context, the role of AEDs may be determinant. In fact, drugs still remain the mainstay of epilepsy treatment but these compounds have a number of mechanisms of 
action which are likely to be responsible not only for their anti-seizure activity but also for their effect on mood and behavior. The psychotropic potential of AEDs is related to direct and indirect mechanisms. ${ }^{20} \mathrm{~A}$ number of studies have suggested that treatment with some AEDs is associated with the occurrence of symptoms of depression while other compounds are probably antidepressants. As far as first generation compounds are concerned, authors agree that there is a link between barbiturates and depression, while carbamazepine probably has beneficial effects on mood. ${ }^{21-23}$ Among second generation AEDs, vigabatrin, ${ }^{24}$ tiagabine,,$^{25}$ and topiramate ${ }^{26}$ have been linked to treatmentemergent depressive symptoms. In some cases, they were associated with a sudden complete control of seizures (the forced normalization phenomenon), ${ }^{27}$ while in others they seemed unrelated to this. In most cases, a rapid titration of the drug ${ }^{28}$ in patients with refractory epilepsy, a past history of depression, ${ }^{29}$ and a limbic system dysfunction ${ }^{29}$ represent major determinants.

\section{AEDs and suicidality: what is the evidence?}

During the last year, a number of retrospective cohort and case-control studies have been published, which have tried to examine the question raised by the FDA meta-analysis using observational study methodology. ${ }^{2,30-36}$ This section of the paper presents methodological considerations and critiques of major studies that are summarized in Table 1. Studies focused only on patients with bipolar disorders have not been included because they are not pertinent to the present discussion. ${ }^{2}$

A major limitation of most of these studies is the failure to adjust for past suicidal behavior. This factor is extremely important because it is a highly recurrent phenomenon (between 14\% and 17\% will repeat in 1 year and more than $30 \%$ over 10 years), ${ }^{37,38}$ and the conditions being studied for which AEDs are prescribed are often associated with suicidal behavior even before diagnosis. ${ }^{39}$ Additionally, some AEDs are thought to have positive effects on mood and others are thought to have negative effects; ${ }^{40}$ thus prescribing practices may be influenced by prior suicidal behavior. In the absence of adjustment for prior suicidal behavior, it is not possible to determine whether or not AEDs are associated with suicidal behavior.

One of the first epidemiological studies to have been published was a Danish nationwide register of prescriptions, comorbidity, psychiatric diagnoses, and cause of death. ${ }^{31}$ Two different approaches were used, a case-crossover study and

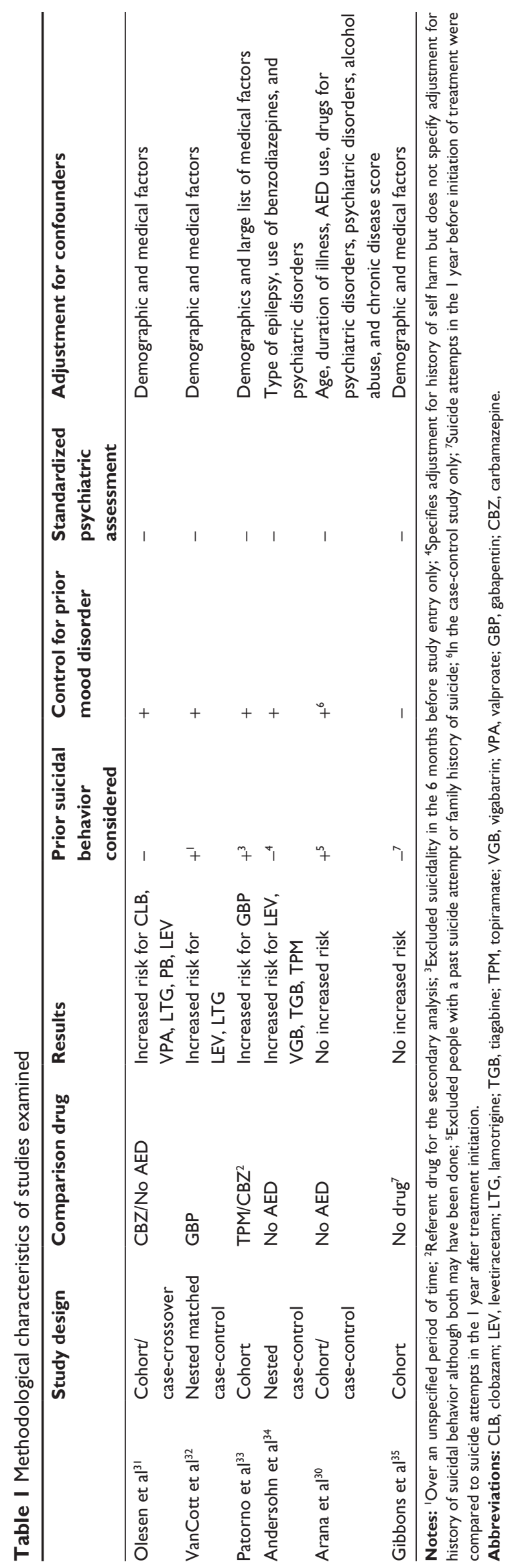


a retrospective cohort study; past suicidal behavior was not accounted for in these studies. In the case-crossover study, cases who committed suicide during a 10-year period during which they had also taken AEDs were compared to themselves during a control period of 30 days 2 (or 4) months before the suicide. This approach is weak as it does not account for suicidality during the control period. The retrospective cohort study included 169,725 people who started taking new AEDs over a 10-year period, and who had not been prescribed AEDs in the year prior to the 10-year period. Suicide during the first 6 months of exposure to a new AED was associated with an increased hazard ratio (compared with carbamazepine) for clonazepam, valproate, lamotrigine, phenobarbital, and levetiracetam. Only a minority of the cohort was registered as having epilepsy and individuals may have restarted AED treatment in response to worsening of underlying psychiatric or pain-related symptoms. Additionally, past suicidality was not assessed. It is interesting that valproate appeared to increase the risk of suicide, which was not the case in the FDA meta-analysis.

Investigators in the US used Veterans Health Administration data to assess suicide-related behaviors in people with a new prescription for monotherapy AED. ${ }^{32}$ For each person aged 66 years and above with new AED treatment who had suicide-related behavior, 12 controls were found, matched according to a previous history of suicide-related behavior, the year of first AED prescription, and a diagnosis of epilepsy. Past suicidality was accounted for over an unspecified time period. Compared with gabapentin (used by more than $75 \%$ of the population), there was a slight increase in suicide-related behaviors in those taking the newer AEDs (levetiracetam or lamotrigine) but only 7 people in the study were taking these drugs and the confidence intervals were wide. The strongest predictor for suicide-related behavior was a diagnosis of an affective disorder prior to the AED prescription. In this study, the FDA's finding that suicidal behavior was more common in those with epilepsy than in those using AEDs for other indications was not confirmed.

Another US cohort study using the HealthCore Integrated Research Database reported an increased risk of suicidal attempts, completed suicide, or violent death in patients taking gabapentin, lamotrigine, oxcarbazepine, tiagabine, and valproate regardless of a specific indication. ${ }^{33}$ People with suicidality in the 6 months before study entry were excluded from the analysis. Only $0.3 \%$ to $19 \%$ of the cohort was taking AEDs for epilepsy and the median follow-up was 60 days. In a secondary analysis (compared to carbamazepine), gabapentin was associated with an increased suicidal risk.
However, this was not seen in the primary analysis with topiramate as the referent where lamotrigine, oxcarbazepine, tiagabine, and valproate conferred and increased risk for suicide attempt or completion. Like other studies in the field, the lack of pre-treatment data on other risk factors for suicidality, including suicidality prior to the 6 months before study entry represents a major limitation.

A nested case-control study used data from the United Kingdom General Practice Research Database (GPRD). ${ }^{34}$ Analysis adjusted for history of self harm but not for history of suicidal behavior. The authors reported a 3-fold increased risk of self harm/suicidal behavior $(\mathrm{OR}=3.08 ; 95 \% \mathrm{CI} 1.22$ to 7.77 ) in patients taking levetiracetam, tiagabine, topiramate, or vigabatrin, arbitrarily classified by the authors themselves as high risk drugs and for this reason analyzed as a group. Findings in this study were based on a very small number of cases ( 2 cases per high risk drug). ${ }^{41}$

Another study adopted two approaches using The Health Improvement Network database, a 5.7\% representative sample of the British General Practice study. They compared the incidence of suicide-related events in groups defined by epilepsy, depression, and AEDs and conducted a case-control study of suicide-related events. ${ }^{30}$ People with a past suicide attempt or with a family history of suicide were excluded, making this the cleanest analysis. The incidence of suiciderelated events per 100,000 person-years was 15.0 (95\% CI 14.6 to 15.5 ) among patients without epilepsy, depression, bipolar disorder, or AED treatment, 38.2 (95\% CI 26.3 to 53.7) among patients with epilepsy who did not receive AEDs, and 48.2 (95\% CI 39.4 to 58.5) among patients with epilepsy who received AEDs. For the case-control study, 5 controls, matched for age, gender, and clinical practice, were selected for each case. In adjusted analyses, the odds of suicide-related events were not increased by AEDs among patients with epilepsy (OR, $0.59 ; 95 \%$ CI 0.35 to 0.98$)$ or bipolar disorder (1.13; 95\% CI 0.35 to 3.61$)$ but were significantly associated among patients with depression (1.65; 95\% CI 1.24 to 2.19) and among those who did not have epilepsy, depression, or bipolar disorder (2.57; 95\% CI 1.78 to 3.71$)$. One possible explanation for these inconsistent results is confounding by indication.

A US study used the PharMetrics medical claims database to investigate the risk of suicide attempt in a cohort of patients taking gabapentin for various indications (epilepsy, pain disorders, bipolar illness, major depression, schizophrenia, and other psychiatric disorders). ${ }^{35}$ Overall, there was no significant difference in suicide attempt rates in the 1 year before versus the 1 year after initiation of gabapentin. 
Pre-prescription suicide attempt rates were 5 times higher in psychiatric populations compared with nonpsychiatric; therefore these groups were analyzed separately. No drug effect was detected in the nonpsychiatric populations (epilepsy and pain syndromes). Significant reductions in suicide attempt rates were seen for bipolar disorder, major depression, or other psychiatric illnesses, presumably because the suicide attempts precipitated initiation of pharmacotherapy.

\section{What's the role of these data in clinical practice?}

As previously stated there seems to be general agreement among authors that risks associated with stopping, or not even starting, AEDs in epilepsy might well be in excess of the so-called risk of suicidality. ${ }^{1,3}$ In fact, the risks associated with not taking AEDs are lack of seizure control and the complications which may ensue such as accidents or Sudden Unexplained Death in Epilepsy (SUDEP). ${ }^{6}$ Data from the RANSOM study suggested that nonadherence to AEDs can have serious or fatal consequences for patients with epilepsy, with a 3-fold increase in mortality risk compared with patients who are adherent to drug treatment. ${ }^{42}$

All studies reviewed in this paper on the relationship between AEDs and suicidality show a lack of concordance and are affected by a number of limitations, ${ }^{43}$ firstly, the lack of a valid standardized psychiatric assessment for suicide. Moreover, data come from sources that have not been developed to study the questions addressed. It has been argued that observational studies of drugs for nonpsychiatric conditions are not suitable for investigating suicide or suicidality. Observational studies may generate hypotheses but are complicated by a number of confounding factors, some of which are not assessed. In clinical trials that use adverse events as the measure of suicidality, suicidal behavior or ideation may not be captured. ${ }^{44}$ However, clinical trials that collect information on suicidality in a standardized fashion at selected time intervals would be ideal to answer these questions, even if these are secondary analyses.

In our opinion, it has to be acknowledged that tailored treatment strategies in epilepsy are mandatory. In particular, clinicians need to pay attention not only to seizure patterns when choosing the appropriate AED but also to a number of different parameters (eg, age, gender, working needs, medical comorbidities, and so on) and not the least the mental state of the patient. In fact, in agreement with others, we suggest that early medical treatment with AEDs could potentially reduce the suicide risk of patients with epilepsy because of the mood-stabilizing properties of some compounds ${ }^{40}$ and the knowledge that suicide attempt and depression can precede the development of epilepsy. ${ }^{39}$ The prognosis of epilepsy and the occurrence of severe complications, including suicide, may be made worse by missing important comorbidity and by delaying or withholding treatment. These issues may be compounded by the lack of input from professionals such as psychologists, social workers, and psychiatrists.

\section{Conclusion}

More than 2 years after the FDA warning on AEDs and suicidality, the scientific community is still far from a final answer to the question. The studies published during the last year generated inconsistent results, attributable to a number of methodological limitations. The FDA will be requiring standardized assessment of suicidality at regular intervals during follow-up in future AED clinical trials. These data will be useful over time in considering whether or not AEDs are associated with suicidal behavior. The FDA alert has definitely increased the awareness of epileptologists on the issue of psychiatric comorbidities in epilepsy, emphasizing the need for standardized approaches for prevention and treatment. Moreover, drug companies are aware that tolerability and safety evaluations during drug development need to take into account behavioral effects. Although it is likely that the question will remain unanswered, this issue has definitely stimulated better tailored treatment strategies and a careful attention to patients' needs.

\section{Disclosure}

The authors have not been paid for the present paper. MM has received travel grants or consultancy fees from various pharmaceutical companies including Pfizer, UCB-Pharma, Eisai, Janssen, and sanofi-aventis, manufacturers of antiepileptic drugs. DCH has received travel grants or consultancy fees from Pfizer and GSK.

\section{References}

1. Bell GS, Mula M, Sander JW. Suicidality in people taking antiepileptic drugs: what is the evidence? CNS Drugs. 2009;23:281-292.

2. Gibbons R, Hur K, Brown CH, Mann JJ. Relationship between antiepileptic drugs and suicide attempts in patients with bipolar disorder. Arch Gen Psychiatry. 2009;66:1354-1360.

3. Hesdorffer DC, Kanner AM. The FDA alert on suicidality and antiepileptic drugs: fire or false alarm? Epilepsia. 2009;50:978-986.

4. Mula M, Bell GS, Sander JW. Suicidality in epilepsy and possible effects of antiepileptic drugs. Curr Neurol Neurosci Rep. 2010;10:327-332.

5. FDA U. Antiepileptic drugs and suicidality. 2008. Available from http:// www.fda.gov/ohrms/dockets/ac/08/briefing/2008-4372b1-01-FDA.pdf. Accessed May 4, 2011

6. FDA U. Information for Healthcare Professionals: Suicidal Behavior and Ideation and Antiepileptic Drugs. 2008 . Available from http://www.fda gov/Drugs/DrugSafety/PostmarketDrugSafetyInformationforPatientsandProviders/ucm100192.htm. Accessed May 4, 2011. 
7. Busco M. FDA Advisory Members Agree Antiepileptics Pose Suicidality Risk, Need for Black-box Warning. http://www.medscape. com/viewarticle/577432.

8. Mula M, Sander JW. Antiepileptic drugs and suicide risk: could stopping medications pose a greater hazard? Expert Rev Neurother. 2010;10: 1775-1776.

9. Kanner AM, Balabanov A. Depression and epilepsy: how closely related are they? Neurology. 2002;58:S27-S39.

10. Edeh J, Toone BK. Antiepileptic therapy, folate deficiency, and psychiatric morbidity: a general practice survey. Epilepsia. 1985;26:434-440.

11. Tellez-Zenteno JF, Patten SB, Jette N, Williams J, Wiebe S. Psychiatric comorbidity in epilepsy: a population-based analysis. Epilepsia. 2007; 48:2336-2344.

12. Victoroff JI, Benson F, Grafton ST, Engel J Jr, Mazziotta JC. Depression in complex partial seizures. Electroencephalography and cerebral metabolic correlates. Arch Neurol. 1994;51:155-163.

13. Ring HA, Moriarty J, Trimble MR. A prospective study of the early postsurgical psychiatric associations of epilepsy surgery. J Neurol Neurosurg Psychiatry. 1998;64:601-604.

14. Jacoby A, Baker GA, Steen N, Potts P, Chadwick DW. The clinical course of epilepsy and its psychosocial correlates: findings from a UK. Community study. Epilepsia. 1996;37:148-161.

15. Harris EC, Barraclough B. Suicide as an outcome for mental disorders. A meta-analysis. Br J Psychiatry. 1997;170:205-228.

16. Bell GS, Gaitatzis A, Bell CL, Johnson AL, Sander JW. Suicide in people with epilepsy: how great is the risk? Epilepsia. 2009;50:1933-1942.

17. Barraclough BM. The suicide rate of epilepsy. Acta Psychiatr Scand. 1987;76:339-345.

18. Christensen J, Vestergaard M, Mortensen PB, Sidenius P, Agerbo E. Epilepsy and risk of suicide: a population-based case-control study. Lancet Neurol. 2007;6:693-698.

19. Hara E, Akanuma N, Adachi N, Hara K, Koutroumanidis M. Suicide attempts in adult patients with idiopathic generalized epilepsy. Psychiatry Clin Neurosci. 2009;63:225-229.

20. Mula M, Sander JW. Negative effects of antiepileptic drugs on mood in patients with epilepsy. Drug Saf. 2007;30:555-567.

21. Rodin EA, Rim CS, Kitano H, Lewis R, Rennick PM. A comparison of the effectiveness of primidone versus carbamazepine in epileptic outpatients. J Nerv Ment Dis. 1976;163:41-46.

22. Dodrill CB, Troupin AS. Psychotropic effects of carbamazepine in epilepsy: a double-blind comparison with phenytoin. Neurology. 1977; 27:1023-1028.

23. Robertson MM, Trimble MR. Depressive illness in patients with epilepsy: a review. Epilepsia. 1983;24(Suppl 2):S109-S116.

24. Levinson DF, Devinsky O. Psychiatric adverse events during vigabatrin therapy. Neurology. 1999;53:1503-1511.

25. Trimble MR, Rusch N, Betts T, Crawford PM. Psychiatric symptoms after therapy with new antiepileptic drugs: psychopathological and seizure related variables. Seizure. 2000;9:249-254.

26. Mula M, Trimble MR, Lhatoo SD, Sander JW. Topiramate and psychiatric adverse events in patients with epilepsy. Epilepsia. 2003;44:659-663.
27. Ring HA, Crellin R, Kirker S, EH. R. Vigabatrin and depression. J Neurol Neurosurg Psychiatry. 1993;56:925-928.

28. Mula M, Hesdorffer DC, Trimble M, Sander JW. The role of titration schedule of topiramate for the development of depression in patients with epilepsy. Epilepsia. 2009;50:1072-1076.

29. Mula M, Trimble MR, Sander JW. Are psychiatric adverse events of antiepileptic drugs a unique entity? A study on topiramate and levetiracetam. Epilepsia. 2007;48:2322-2326.

30. Arana A, Wentworth CE, Ayuso-Mateos JL, Arellano FM. Suiciderelated events in patients treated with antiepileptic drugs. $N$ Engl J Med. 2010;363:542-551.

31. Olesen JB, Hansen PR, Erdal J, et al. Antiepileptic drugs and risk of suicide: a nationwide study. Pharmacoepidemiol Drug Saf. 2010;19: 518-524.

32. VanCott AC, Cramer JA, Copeland LA, et al. Suicide-related behaviors in older patients with new anti-epileptic drug use: data from the VA hospital system. BMC Med. 2010;8:4

33. Patorno E, Bohn RL, Wahl PM, et al. Anticonvulsant medications and the risk of suicide, attempted suicide, or violent death. JAMA. 2010;303: 1401-1409.

34. Andersohn F, Schade R, Willich SN, Garbe E. Use of antiepileptic drugs in epilepsy and the risk of self-harm or suicidal behavior. Neurology. 2010;75:335-340.

35. Gibbons RD, Hur K, Brown CH, Mann JJ. Gabapentin and suicide attempts. Pharmacoepidemiol Drug Saf. 2010;19:1241-1247.

36. Redden L, Pritchett Y, Robieson W, et al. Suicidality and divalproex sodium: analysis of controlled studies in multiple indications. Ann Gen Psychiatry. 2011;10:1.

37. Kapur N, Cooper J, King-Hele S, et al. The repetition of suicidal behavior: a multicenter cohort study. J Clin Psychiatry. 2006;67:1599-1609.

38. Owens D, Horrocks J, House A. Fatal and non-fatal repetition of self-harm. Systematic review. Br J Psychiatry. 2002;181:193-199.

39. Hesdorffer DC, Ludvigsson P, Hauser WA, Olafsson E, Kjartansson O. Co-occurrence of major depression or suicide attempt with migraine with aura and risk for unprovoked seizure. Epilepsy Res. 2007;75: 220-223.

40. Ettinger AB. Psychotropic effects of antiepileptic drugs. Neurology. 2006;67:1916-1925.

41. Mula M, Sander JW. Antiepileptic drugs and suicidality. Much ado about very little? Neurology. 2010;75:300-301.

42. Faught E, Duh MS, Weiner JR, Guérin A, Cunnington MC. Nonadherence to antiepileptic drugs and increased mortality: findings from the RANSOM Study. Neurology. 2008;71:1572-1578.

43. Hesdorffer DC, Berg AT, Kanner AM. An update on antiepileptic drugs and suicide: are there definitive answers yet? Epilepsy Curr. 2010;10: $137-145$.

44. Reith DM, Edmonds L. Assessing the role of drugs in suicidal ideation and suicidality. CNS Drugs. 2007;21:463-472.
Drug, Healthcare and Patient Safety

\section{Publish your work in this journal}

Drug, Healthcare and Patient Safety is an international, peer-reviewed open-access journal exploring patient safety issues in the healthcare continuum from diagnostic and screening interventions through to treatment, drug therapy and surgery. The journal is characterized by the rapid reporting of reviews, original research, clinical, epidemiological and

\section{Dovepress}

post-marketing surveillance studies, risk management, health literacy and educational programs across all areas of healthcare delivery. The manuscript management system is completely online and includes a very quick and fair peer-review system. Visit http://www.dovepress.com/ testimonials.php to read real quotes from published authors. 